\title{
MicroRNA-323a-3p Promotes Pressure Overload-Induced Cardiac Fibrosis by Targeting TIMP3
}

\author{
JingChao Zhang ${ }^{a}$ Yan Lang ${ }^{b}$ LongHui Guo ${ }^{a} \quad$ Yu Pei $^{a} \quad$ Shuang Hao \\ ZhenXing Liang ${ }^{\mathrm{a}}$ Gang Su ${ }^{\mathrm{a}}$ LiLiang Shu ${ }^{\mathrm{a}}$ Hai Liu ${ }^{\mathrm{a}}$ Chen Huang ${ }^{\mathrm{a}}$ Jing $\mathrm{Xu}^{\mathrm{a}}$ \\ aDepartment of Cardiovascular Surgery, The First Affiliated Hospital of Zhengzhou University, \\ Zhengzhou, 'Department of Psychiatry, The First Affiliated Hospital of Zhengzhou University, \\ Zhengzhou, China
}

\section{Key Words}

miR-323-3p • TAC • Cardiac fibrosis • TIMP3 • TGF $\beta$

\begin{abstract}
Background/Aims: Cardiac fibrosis is a major cause of diverse cardiovascular diseases. MicroRNAs have recently been proven a novel class of regulators of cardiac fibrosis. In this study, we sought to investigate the role of miR-323a-3p and its mechanisms in regulating cardiac fibrosis. Methods: The transverse aortic constriction (TAC) mice model was induced and neonatal cardiac fibroblasts (CFs) were cultured. MTT (3- [4, 5-dimethylthiazol-2-yl]-2, 5-diphenyltetrazolium bromide) assay was used to detect the cell viability. Echocardiography was used to evaluate cardiac function. Masson's Trichrome stain was used to evaluate the development of fibrosis. Luciferase activity assay was performed to confirm the miRNA's binding site. Real-time PCR and Western blot were used to evaluate the level of mRNA and protein. Results: MiR-323a-3p was found up-regulated in myocardial tissues subjected to TAC and in CFs cultured with Angiotensin II (Ang II). Overexpression of miR-323a-3p significantly increased the mRNA levels of collagen I, collagen III, MMP2 and MMP9, while inhibition of miR-323a-3p prevented the proliferation, collagen production and the protein level of transforming growth factor (TGF- $\beta$ ) in rat neonatal CFs. Strikingly, injection of antagomiR$323 a-3 p$ elevated cardiac function and inhibited the expression of TGF- $\beta$ in the TAC mice. TIMP3 was a direct target of miR-323a-3p, as the overexpression of miR-323a-3p decreased the protein and mRNA levels of TIMP3. In the CFs with pre-treatment of Ang II, siRNATIMP abolished the effects of AMO-323a-3p on the inhibition of the proliferation of CFs, the down-regulation of collagen I and collagen III, and the expression of TGF- $\beta$. Conclusion: Our findings provide evidence that miR-323a-3p promotes cardiac fibrosis via miR-323a-3pTIMP3-TGF- $\beta$ pathway. miR-323a-3p may be a new marker for cardiac fibrosis progression and that inhibition of miR-323a-3p may be a promising therapeutic target for the treatment of cardiac fibrosis.

J. Zhang and Y. Lang contributed equally to this work.




\section{Cellular Physiology Cell Physiol Biochem 2018;50:2176-2187

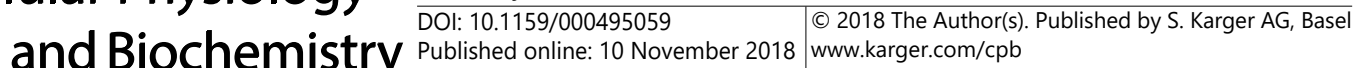

\section{Introduction}

Cardiac fibrosis is an important contributor to the development of various cardiovascular diseases, including myocardial infarction, atrial brillation and pressure overload-induced cardiac hypertrophy, ultimately leading to reduced cardiac diastolic and contractile function that may progress to heart failure [1-3]. Fibrosis is characterized by an abnormal balance of extracellular matrix (ECM) proteins, such as collagen I, collagen III and matrix metalloproteinases (MMPs) [1, 4]. It is a complex process resulting from activation of a wide variety of signaling pathways. Although improvements in treatment strategies have been provided during the past decade, no efficient therapeutic approaches are available for the treatment of fibrosis. Therefore, it is critical to identify and characterize key factors that regulate myocardial fibrosis and reverse the damage to the heart.

MicroRNAs (miRNAs) are endogenous, single-stranded, 20-23-nucleotide noncoding RNAs that act as negative regulators of gene expression by promoting the degradation or inhibiting the translation of target mRNAs. Growing evidence has demonstrated that miRNAs play important roles in the regulation of development, differentiation, proliferation, and apoptosis [5, 6]. Notably, it has been reported that several miRNAs contribute to the development and progression of cardiac fibrosis [7-9]. For example, up-regulation of miR21 [10], miR-34a and miR-93 [11] promoted the development of cardiac fibrosis, whereas overexpression of miR-133, and miR-590 [12], together with down-regulation of miR-23 [13], miR-29 [14], miR-101 [15] and miR-132 [16], suppressed the fibrotic responses of cardiac fibroblasts (CFs). Recently, aberrant expression of miR-323 has been detected in various human diseases. MiR-323a-3p plays central roles in cancers $[17,18]$. MiR-323a-3p is an important factor in cerebral ischemic injury [19]. Moreover, miR-323a-3p has been demonstrated to be a candidate marker for the diagnosis of cardiomyopathy in Friedreich's ataxia patients and the presence of acute coronary syndromes [20,21]. However, the probably role and precise mechanism of miR-323a-3p in the process of cardiac fibrosis remains unclear.

In this study, we aimed to investigate the effects of miR-323a-3p on cardiac fibrosis using a mice model of transverse aortic constriction (TAC) and a cellular model induced by Angiotensin II (Ang II). We hypothesized that miR-323a-3p promoted the proliferation of CFs and enhanced the expression of ECM proteins. Inhibition of miR-323a-3p by its specific antisense inhibitor was able to attenuate cardiac fibrosis and improve cardiac function. Our results further showed that miR-323a-3p promotes cardiac fibrosis through the targeted induction of TIMP3-TGF- $\beta$ pathway. This study implies that manipulating the expression of miR-323a-3p represents a promising therapeutic strategy for the treatment of cardiac fibrosis.

\section{Materials and Methods}

Mice model of transverse aortic constriction (TAC)

Healthy male C57BL/6 mice aged 8-10 weeks and weighing 20-25 g were used in the study. To make the cardiac pressure overload model, animals were subjected to transverse aortic constriction (TAC). Briefly, the mice were anesthetized with pentobarbital sodium ( $6 \mathrm{mg} \bullet \mathrm{kg}^{-1}$, i.p.) until they were unresponsive to toe pinching. Then, they were orally intubated and placed on a ventilator for artificial respiration. After the chest was opened, a 7-0 silk suture was placed around the transverse aorta and tied around a 26-gauge blunt needle, which was immediately removed after ligation. Sham group animals were subjected to the same open chest procedures without aorta constriction. All the animals were kept ventilated until recovery of autonomic breath. During the experiment, mice were kept on a $12 \mathrm{~h}$ dark-light cycle. The animals were kept under standard animal room conditions with temperature $20 \pm 1^{\circ} \mathrm{C}$, humidity $55-60 \%$ and free access to food and water. 


\section{Cellular Physiology Cell Physiol Biochem 2018;50:2176-2187 \begin{tabular}{l|l|l|l|l}
\hline DOl: 10.1159/000495059 & $\begin{array}{l}\text { C } 2018 \text { The Author(s). Published by S. Karger AG, Basel } \\
\text { www.karger.com/cpb }\end{array}$
\end{tabular}

All animal experiments were approved by the Committee on Animal Experimentation of Zhengzhou University and were conducted in accordance with international guidelines regarding animal experimentation.

\section{Animal Experiments}

The cholesterol-conjugated miR-323a-3p antisense and negative control (antagomiR-323a-3p and antagomiR-NC, respectively) were purchased from RiboBio (Guangzhou, China). Mice were randomly assigned to four groups prior to TAC surgery: Sham, TAC, TAC with antagomiR-NC, and TAC with antagomiR323a-3p. After TAC operation, antagomiR-323a-3p (30 mg/kg) and antagomiR-NC (30 mg/kg) were injected one time a day by tail vein for fourteen consecutive days. The Sham and TAC groups were fed orally with equal volumes of physiological saline once daily. After twenty-eight days, we evaluate their cardiac function and measure the fibrotic factors using heart tissue.

\section{Echocardiographic measurements}

Transthoracic echocardiography was performed on mice at baseline (day 0), day 28 post-surgery by using an ultrasound machine (Vivid 7, GE Medical, Horten, Norway) equipped with a 10-MHz phased-array transducer. Mice were sedated with isoflurane (2.5\% isoflurane for induction and $0.5 \%$ for maintenance) and placed in the left lateral decubitus position. LV mass and functional parameters such as the left ventricular ejection fraction (EF) and fractional shortening (FS) were calculated from the M-mode recording. All the measurements were averaged from at least three separate cardiac cycles. After functional measurement, the hearts of mice were excised, the myocardial tissue samples were rapidly frozen in liquid nitrogen for subsequent western blot or fixed in $4 \%$ paraformaldehyde for histological analysis.

\section{Histological analysis}

Myocardial tissue samples fixed in 4\% paraformaldehyde were embedded with paraffin and cut into 5 $\mu \mathrm{m}$-thick slices according to standard histological protocols. Sections were stained with Masson trichrome for the evaluation of cardiac fibrosis. Quantitative measurement of the fibrotic areas was calculated with image analysis software (Image-Pro Plus 6.0 software) and expressed as a percentage of the total surface area of the tissue section.

\section{Cardiac fibroblasts (CFs) isolation and culture}

Neonatal rat cardiac fibroblasts (CFs) were isolated from 1- to 3-day-old Sprague-Dawley (SD) rats. Hearts were minced and placed together in $0.25 \%$ trypsin. Pooled cell suspensions were centrifuged and resuspended in Dulbecco-modified Eagle medium (Hyclone) containing 10\% fetal bovine serum, $100 \mathrm{U} / \mathrm{ml}$ penicillin and $100 \mu \mathrm{g} / \mathrm{ml}$ streptomycin under standard culture conditions ( $37^{\circ} \mathrm{Cin} 5 \% \mathrm{CO}$, 95\% air). CFs were isolated by removal of myocytes through selective adhesion of non-myocytes at a 1-2 h pre-plating interval. CFs were passaged when the cell confluence achieved $70-80 \%$ and the second or third passages were used in our experiment. In addition, CFs were starved for $24 \mathrm{~h}$ in serum-free medium before treatment.

\section{Transfection}

MiR-323a-3p, NC, AM0-323a-3p, siRNA-TIMP3 and siRNA-NC were synthesized by GenePharma (Shanghai, China). CFs were transfected with these RNAs using Lipofectamine 2000 (Invitrogen) following the manufacturer's instruction at a final concentration of $50 \mathrm{nM}$. After $6 \mathrm{~h}$, the transfected CFs were treated with Ang II (100 nM, Sigma-Aldrich) in serum-free medium for $24 \mathrm{~h}$ to induce cardiac fibrosis in vitro.

\section{MTT assay}

3-(4, 5-dimethylthiazol-2-yl)-2, 5-diphenyltetrazolium bromide (MTT) cell vitality assay was used to evaluate CFs proliferation. Firstly, CFs were seeded onto 96-well plates and cultured for $48 \mathrm{~h}$. Following culturing in serum-free medium for $24 \mathrm{~h}$, Ang II, either alone or in combination with AM0-323a-3p (100 $\mathrm{nM}$ ) were added to each well, and incubated with the cells for $48 \mathrm{~h}$. The CFs were then treated with MTT (5 $\mathrm{mg} / \mathrm{mL}$ ) at $37^{\circ} \mathrm{C}$ for $4 \mathrm{~h}$ and absorbance was measured at $570 \mathrm{~nm}$ using a microplate reader. 


\section{Cellular Physiology Cell Physiol Biochem 2018;50:2176-2187

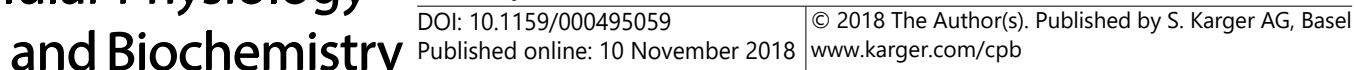

Quantitative reverse transcription-PCR ( $q R T-P C R)$

Total RNA from cultured CFs was isolated using Trizol reagent (Invitrogen) and reverse-transcribed using high-capacity cDNA reverse transcription kit (Applied Biosystems, USA). The mRNA levels of collagen I, collagen III , MMP2, MMP9, and TIMP3 were performed using SYBR Green I incorporation method on ABI 7500 fast Real-Time PCR system (Applied Biosystems), with GAPDH as an internal control. The forward and reverse PCR oligonucleotide primers are listed in Table 1 . The relative mRNA level was determined as values of $2^{-\Delta \Delta C t}$. The level of miR-323a-3p was tested using TaqMan MicroRNA Assay Kit (Applied Biosystems), and normalized to U6 level in each sample.

\section{Western blot analysis}

Total protein was extracted from either the cultured CFs or the myocardial tissues, and the concentration of the proteins was determined using a BCA Protein Assay Kit (Beyotime, Shanghai, China). Equal concentrations of proteins were resolved by $10 \%$ SDS-PAGE and were later transferred onto PVDF membranes. After blocking with $5 \%$ non-fat dried milk in TTBS $(0.1 \%$ Tween- 20$)$ for $1 \mathrm{~h}$, the membranes were incubated with primary antibodies including TIMP3 (1:1, 000 dilution, Abcam, UK), TGF- $\beta$ (1:200 dilution, Santa Cruz, USA), and GAPDH (1:500 dilution, Zhongshanjinqiao, China) at $4^{\circ} \mathrm{C}$ overnight. Membranes were then incubated with a secondary antibody (1:10, 000, Alexa Fluor IRDye 800, Molecular Probes, Eugene USA) at room temperature for $1 \mathrm{~h}$. Images were obtained using an Odyssey Infrared Imaging System (LI-COR Biosciences, USA) and quantified with odyssey v1.2 software. The results were expressed as fold changes by normalizing the data to the control values.

\section{Luciferase assay}

For luciferase assays, the HEK293 cells were cultured in 24-well culture plates and co-transfected with wild-type or mutant TIMP3 3 '-UTR reporters $(0.1 \mu \mathrm{g})$ and AMO-323a-3p expressing plasmid or empty vector $(1 \mu \mathrm{g})$. Luciferase activities were measured at $48 \mathrm{~h}$ after transfection with the application of the Dualluciferase Reporter Assay System (Promega), according to the manufacturer's instructions.

\section{Statistical analysis}

All data were analyzed using the SPSS 17.0 software and were expressed as mean \pm S.E.M. Differences between two groups were evaluated by an unpaired, two-tailed Student t-test, and for multiple comparisons, it was determined using one-way ANOVA followed by Tukey's post-test. A value of $P<0.05$ was considered statistically significant.

\section{Results}

Up-regulation of miR-323a-3p in TAC hearts and Ang II treated rat CFS

The expression of miR-323a-3p was first detected in TAC hearts and cultured CFs treated with Ang II. As shown in Fig. 1A, twenty-eight days following TAC, significant up-regulation of miR-323a-3p expression was observed in TAC hearts compared with sham-operated animals. MiR-323a-3p expression was also higher in cultured CFs with the treatment of 100 nM Ang II than non-treated control cells (Fig. 1B). These findings suggested the possible involvement of miR-323a-3p in CFs proliferation. Intriguingly, TIMP3 protein expression was found consistently down-regulated in both TAC hearts and CFs treated with Ang II (Fig. $1 \mathrm{C}$ and D), which was opposite to the changes of miR-323a-3p.
Table 1. The sequences of primers designed

\begin{tabular}{lc}
\hline Name & Primer Sequence $\left(5^{\prime}\right.$ - $\left.^{\prime}\right)$ \\
\hline Collagen I & F: AAGAAGACATCCCTGAAGTCA \\
R: TTGTGGCAGATACAGATCAAG & F: TTGGGATGCAGCCACCTTG \\
Collagen III & R: CGCAAAGGACAGATCCTGAG \\
MMP2 & F: GGGTCTCAGAACGCCGTGGAG \\
& R: ACAGGACGCAGAGAACCCGC \\
MMP9 & F: GGGCATCTGGGGATTGAACTCAGC \\
& R: AGCGCCCGACGCACAGTAAG \\
TIMP3 & F: ACTGCAAGATCAAGTCCTGCT \\
& R: AGGCGTAGTGTTTGGACTGG \\
GAPDH & F: AAGAAGGTGGTGAAG CAGGC \\
& R: TCCACCACCCAGTTGCTGTA \\
\hline
\end{tabular}


MiR-323a-3p promoted the proliferation of CFs

CFs were transfected with miR-323a-3p (50 nM) and AM0-323a-3p (100 nM) to overexpress and knock down miR-323a-3p, respectively. At $48 \mathrm{~h}$ post-transfection, miR-323a-3p levels were measured by quantitative RTPCR. Transfection with miR323a-3p augmented miR$323 a-3 p$ expression, whereas transfection with AMO-323a$3 p$ reduced miR-323a-3p levels significantly (Fig. 2A, B). We further investigated the effects of miR-323a-3p on the mRNA levels of the ECM protein collagen I, collagen III, MMP2 and MMP9. Compared with negative control group, overexpression of miR-323a$3 p$ significantly increased the mRNA levels of collagen I, collagen III, MMP2 and MMP9 (Fig. 3A-D), while AMO323a-3p, a specific inhibitor of miR-323a-3p, significantly decreased the mRNA expression of collagen I, collagen III, MMP2 and MMP9 (Fig. 3E-H).

The effects of miR-323a-3p on neonatal $\mathrm{CFs}$ proliferation were analyzed by MTT assay. Treatment with $100 \mathrm{nM}$ Ang II on CFs led to a significant increase in CFs proliferation, whereas transfection with AMO-323a$3 p$ markedly attenuated Ang II-induced proliferation of CFs (Fig. 4A). Similarly, the mRNA levels of collagen I, collagen III, MMP2 and MMP9, together with the protein level of the cardiac fibrosis-associated protein TGF- $\beta$ were also significantly up-regulated with the treatment of Ang II (100 nM). Transfection with AMO-323a-3p suppressed Ang II-induced collagen I, collagen III, MMP2, MMP9 and TGF- $\beta$ up-regulation (Fig. 4B-F).

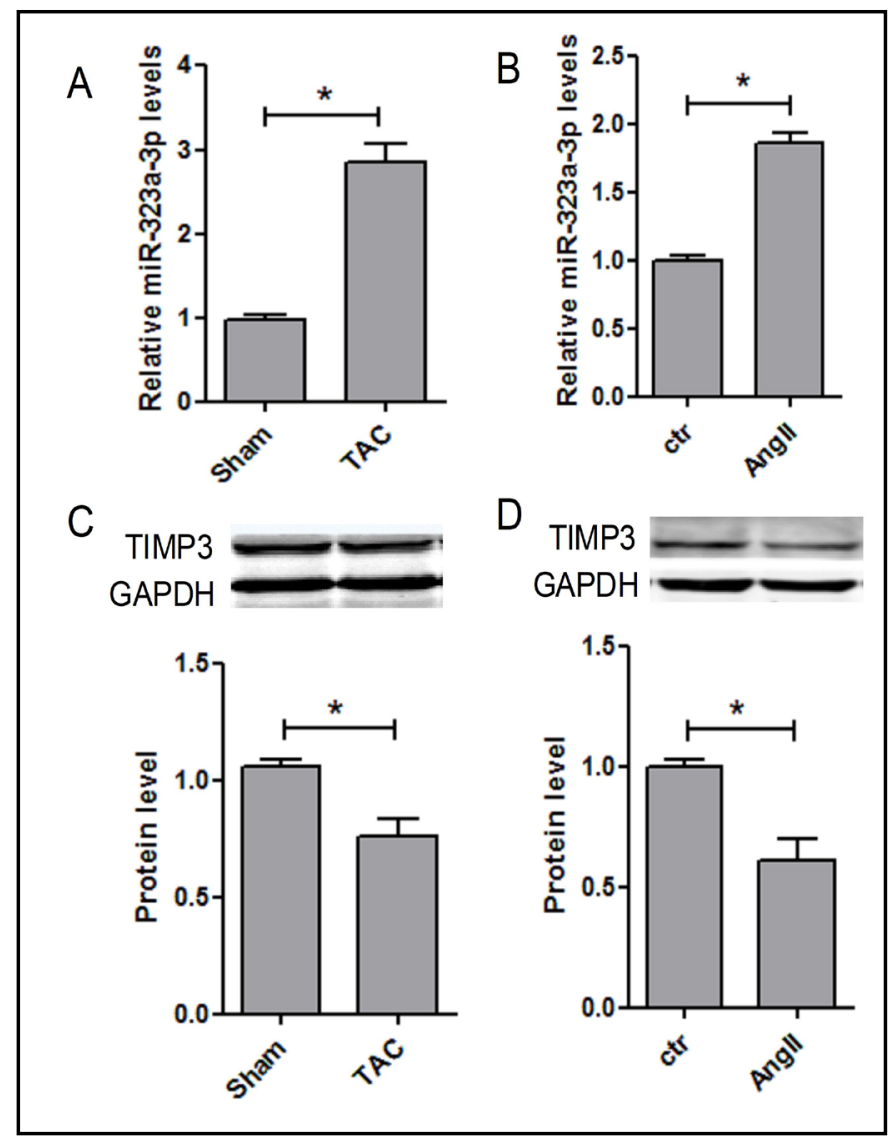

Fig. 1. Expression levels of miR-323a-3p. (A) The up-regulated expression of miR-323a-3p in the hearts underwent transverse aortic constriction (TAC) $(n=12)$. (B) The up-regulated expression of miR-323a-3p in cultured cardiac fibroblasts (CFs) treated with Ang II $(n=8)$. (C) Protein expression levels of TIMP3 in the hearts underwent TAC $(n=12)$. (D) Protein expression levels of TIMP3 in CFs treated with Ang II $(\mathrm{n}=8),{ }^{*} \mathrm{P}<0.05$.

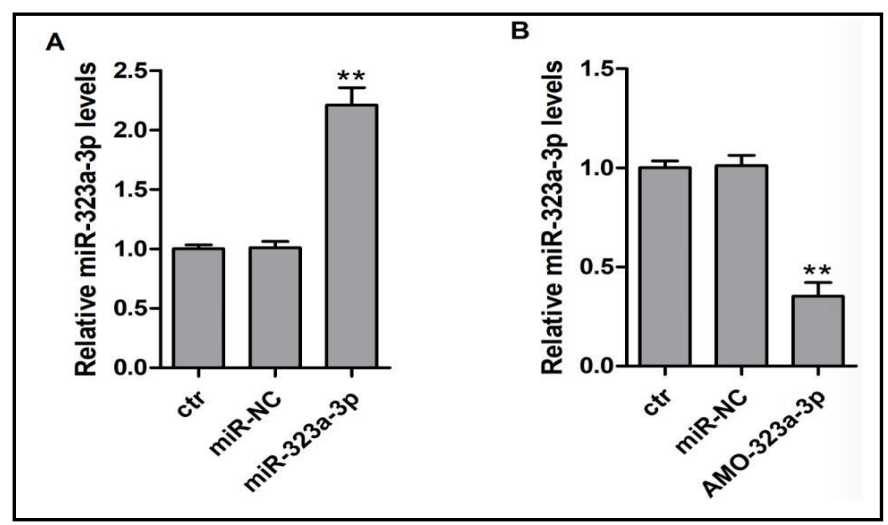

Fig. 2. Overexpression or knockdown of miR-323a-3p in CFs. (A) Transfection of miR-323a-3p (50 nM) in CFs significantly increased the expression of miR-323a-3p, as determined by quantitative real-time PCR. (B) Transfection of antagomir-323a-3p (100 nM) in CFs significantly decreased the expression of miR-323a-3p (n $=5$, mean $\pm \mathrm{SEM}$, ${ }^{* *} \mathrm{P}<0.05$, vs. NC). NC indicates negative control. 


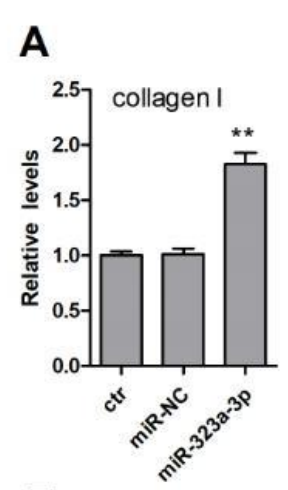

E

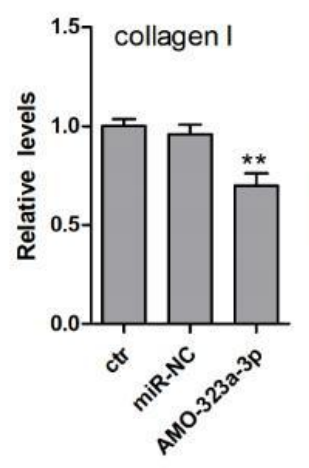

B

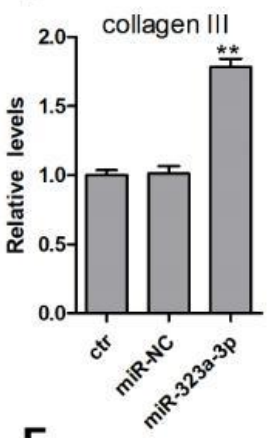

$\mathbf{F}$

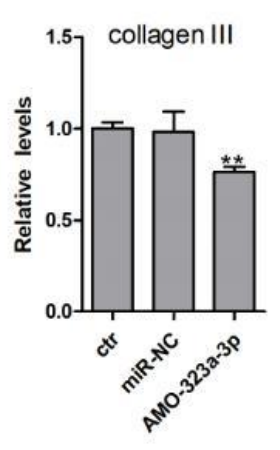

C

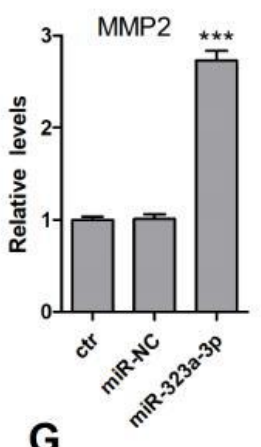

G

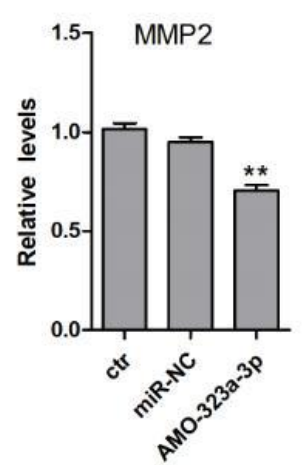

D

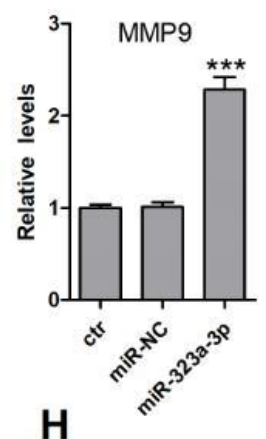

H

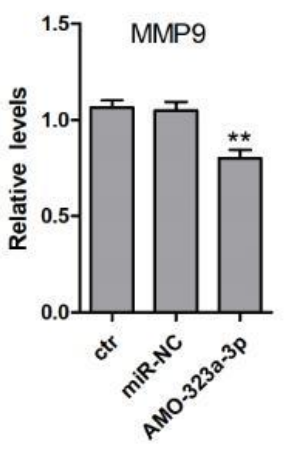

Fig. 3. Transfection of miR-323a-3p (50 nM) increased the mRNA levels of collagen I (A), collagen III (B), MMP2 (C) and MMP9 (D). Transfection of antagomir-323a-3p (100 nM) decreased the mRNA levels of collagen I (E), collagen III (F), MMP2 (G) and MMP9 (H). ( $\mathrm{n}=5$, mean $\pm \mathrm{SEM},{ }^{* *} \mathrm{P}<0.05,{ }^{* * *} \mathrm{P}<0.001$ vs. NC).

Fig. 4. Fibrotic effects of miR-323a3p. (A) MTT assay de monstrated that AMO-323a$3 p$ inhibited the proliferation of CFs treated with Ang II $(100 \mathrm{nM})$. (B-E) AMO-323a$3 p$ decreased the mRNA levels of collagen I, collagen III, MMP2 and MMP9 in CFs treated with Ang II by qRTPCR. (F) Western blot analysis was performed to

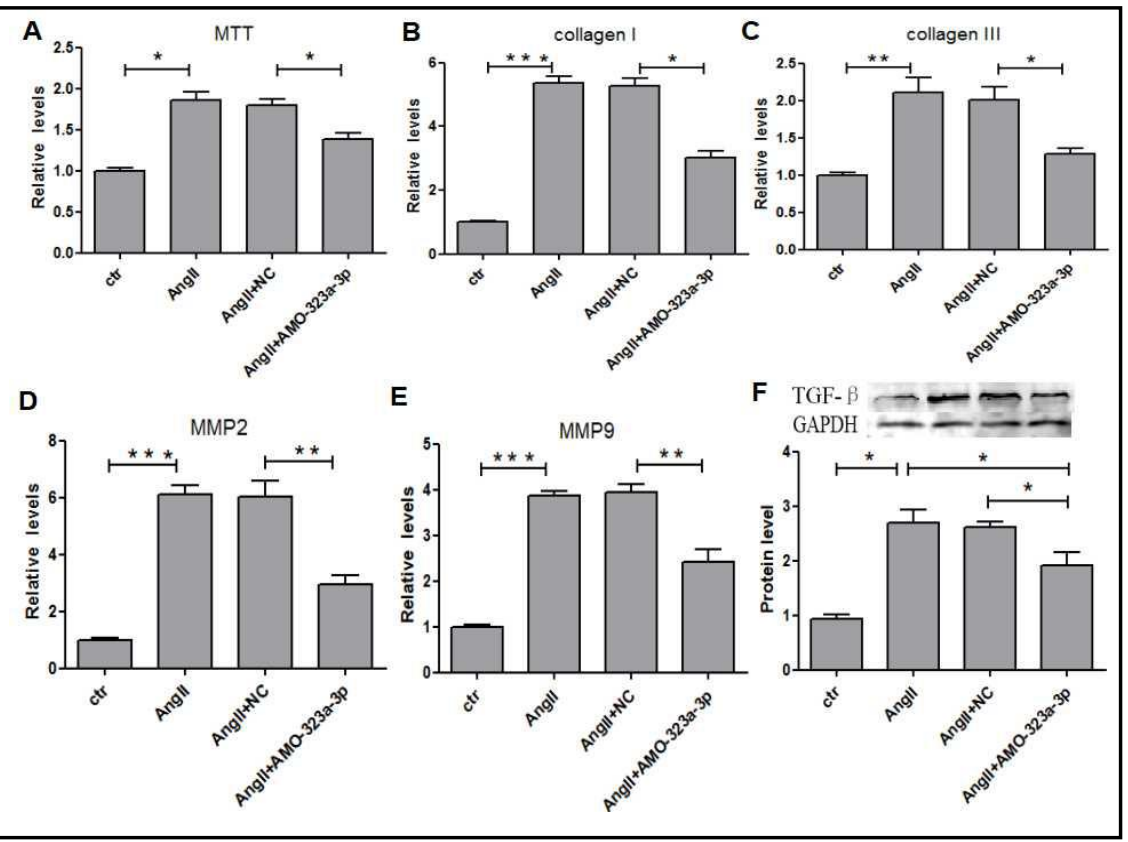
determine AMO-

323a-3p reduced the protein level of TGF- $\beta$ in CFs treated with Ang II. Data are expressed as the means \pm S.E.M.; $\mathrm{n}=5-6$. $\mathrm{NC}$ indicates negative control. ${ }^{*} \mathrm{P}<0.05,{ }^{* *} \mathrm{P}<0.01,{ }^{* * *} \mathrm{P}<0.001$. 


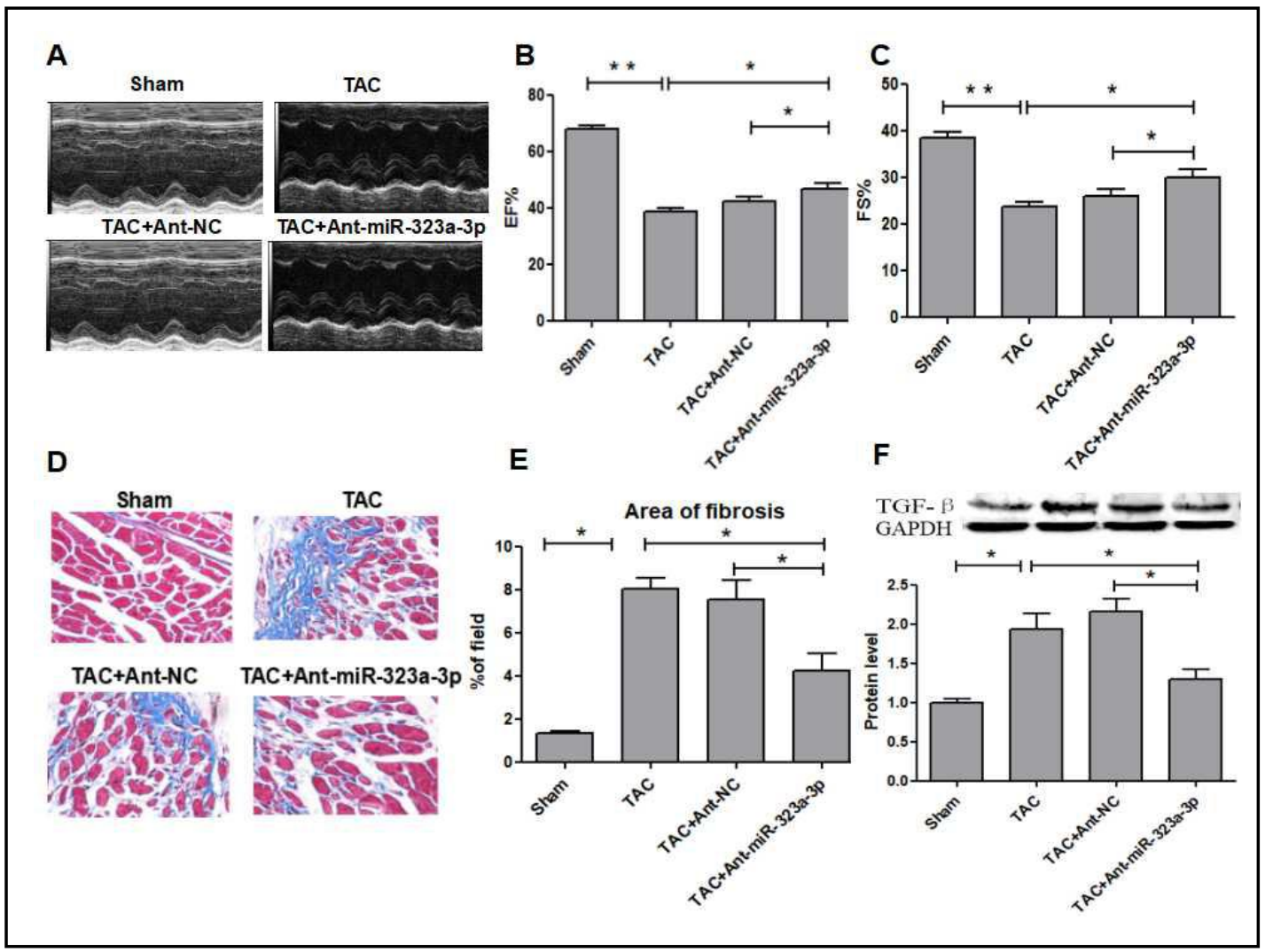

Fig. 5. Inhibition of AMO-323a-3p on TAC-induced cardiac fibrosis. (A) M-mode echocardiographic photos. (B and C) EF\% and FS\% levels evaluated by echocardiography ( $n=7)$. (D) Representative sections of heart with Masson's trichrome staining at a magnification of $200 \times$. The fibrotic tissues are stained blue and the viable tissues red. (E) Effects of antagomiR-323a-3p on area of fibrosis measured from the Masson trichrome-stained sections. Collagen deposition was quantified with an automated image analyzer and expressed as percentage of tissue area. (F) Effects of antagomiR-323a-3p on the protein levels of TGF- $\beta$. Data are expressed as the means \pm S.E.M.; $\mathrm{n}=5-6 .{ }^{*} \mathrm{P}<0.05 ;{ }^{* *} \mathrm{P}<0.01$.

\section{Inhibition of AMO-323a-3p on TAC-induced cardiac fibrosis}

To further confirm the role of miR-323a-3p in cardiac fibrosis development, we next investigated whether administration of AMO-323a-3p could decrease TAC-induced cardiac fibrosis using an in vivo experiment. After TAC surgery, we injected the AMO-323a-3p 30 $\mathrm{mg} / \mathrm{kg}$ one time a day by tail vein for fourteen days. At twenty-eight days post-surgery, we evaluated their cardiac function by echocardiography and measured the fibrotic factors using heart tissue. As shown in Fig. 5A-C, decreased EF\% and FS\% were prevented in the AMO-323a-3p-treated group, compared with the TAC group. These results demonstrated that the AMO-323a-3p could improve the cardiac function induced by TAC. ECM deposition was assessed using Masson's trichrome staining of cardiac tissue sections. The fibrotic areas of TAC mice significantly increased in comparison with sham controls. Consistent with the above results, transfection of AMO-323a-3p significantly alleviated ECM deposition, while the control antagomir showed no effects (Fig. 5D-E). Western Blotting results showed that AMO-323a-3p down-regulated the expression of TGF- $\beta$, compared to the TAC group (Fig. 5F). 


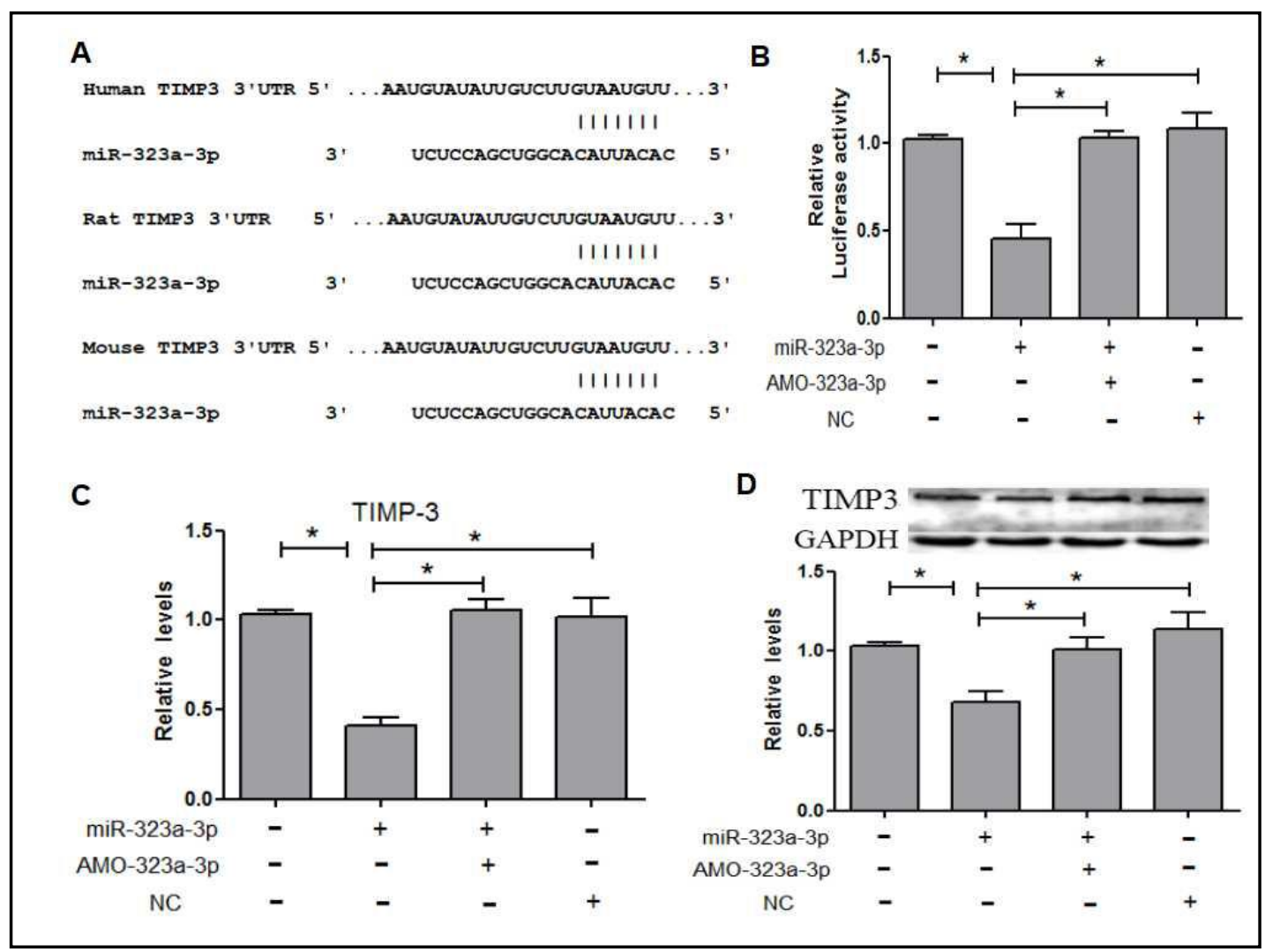

Fig. 6. TIMP3 is a target of miR-323a-3p. (A) Predicted miR-323a-3p target sequences in the $3^{\prime}$ UTR of TIMP3 among mouse, rat, and human. (B) The luciferase reporter assay results depicting the activities of chimeric vectors carrying the luciferase gene and a fragment of the TIMP3 3'-UTR from rats containing the binding sites of miR-323a-3p. (C) was the mRNA and (D) was the protein level of TIMP3 induced by the overexpression of miR-323a-3p in cultured neonatal rat CFs. Data are expressed as the means \pm S.E.M.; $n=$ 4. NC indicates negative control. $* \mathrm{P}<0.05$.

\section{TIMP3 is a target of miR-323a-3p and TIMP3 modulates TGF- $\beta$ expression in CFs}

As described in the earlier section, miR-323a-3p and TIMP3 demonstrated reciprocal changes in their levels in TAC hearts and cultured CFs treated with Ang II, indicating a targeting relationship between these two molecules. Our computational analysis predicted that miR-323a-3p had the potential to suppress TIMP3: the 3'UTR of TIMP3 mRNA was a direct target of miR-323a-3p (Fig. 6A). In this study, we confirmed the regulation of TIMP3 by miR-323a-3p using the luciferase reporter assay. We found that miR-323a-3p (50 nM) significantly inhibited the activity of luciferase vectors containing the 3'UTR of TIMP3 mRNA, which was rescued by co-application of AM0-323a-3p (100 nM) to knockdown miR-323a$3 p$. The specificity of miR-323a-3p action was determined by the absence of changes with the use of NC-a negative control (Fig. 6B).

We then determined if the expression of TIMP3 was affected by the overexpression of miR-323a-3p. Our data indicated that the mRNA and protein level of TIMP3 were decreased by transfection with miR-323a-3p, which was antagonized by co-transfection of miR-323a3p with AM0-323a-3p (Fig. 6C-D).

Next, to further confirm the role played by TIMP3 in miR-323a-3p-mediated cardiac fibrosis, we transfected AMO-323a-3p (100 nM) into CFs treated with siRNA-TIMP3 (50 $\mathrm{nM})$ and siRNA-NC (50 $\mathrm{nM}$ ) respectively. As shown in Fig. 7A-C, siRNA-TIMP3 abolished the inhibition of the proliferation of CFs, the down-regulation of collagen I and collagen III induced by AMO-323a-3p in the cultured CFs with the pre-treatment of Ang II (100 nM). We then detected the protein level of TGF- $\beta$, and found AMO-323a-3p inhibited the up-regulation 


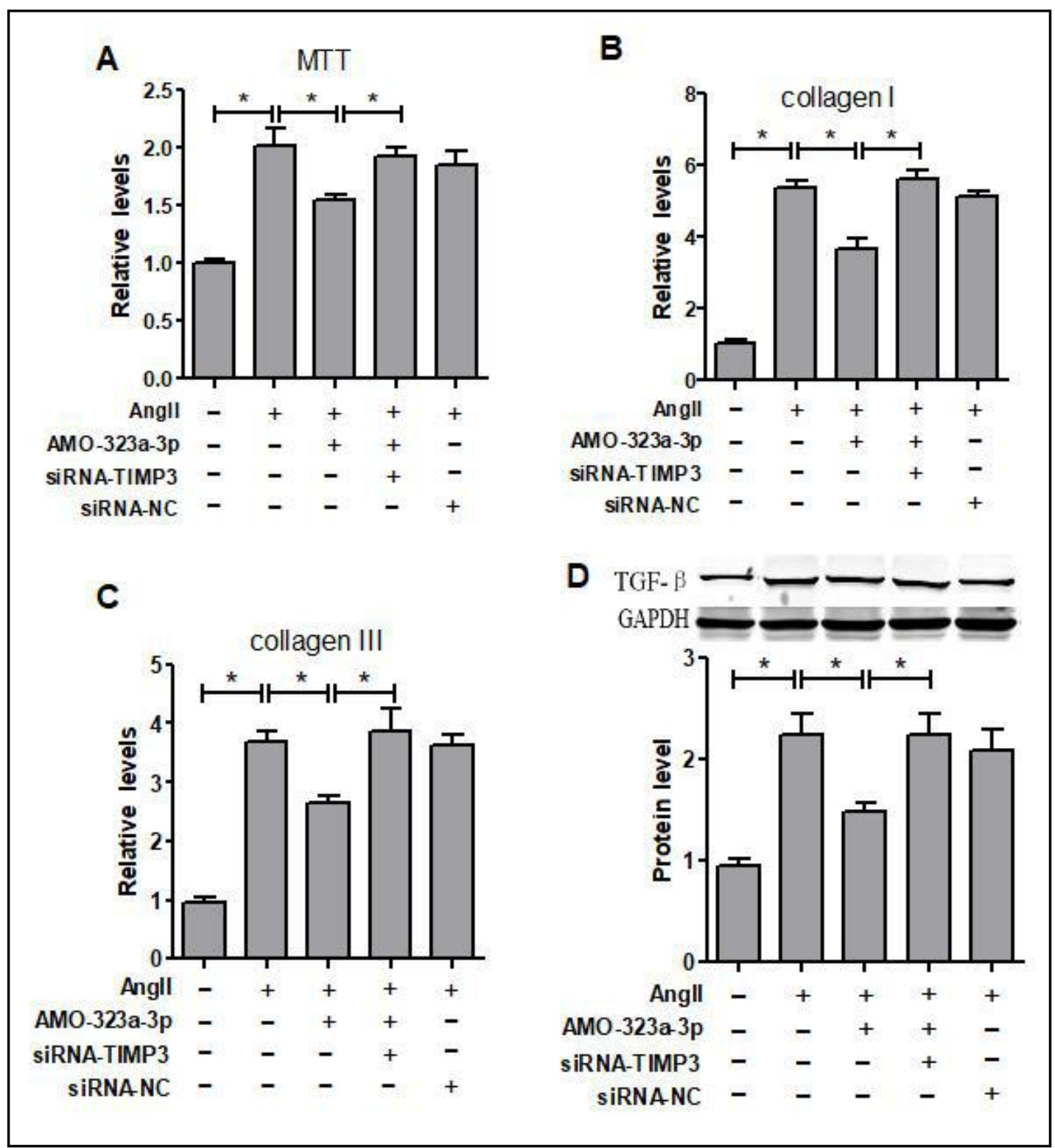

Fig. 7. TIMP3 modulates the fibrotic effects of miR-323a-3p in a TGF- $\beta$ dependent pathway in CFs. SiRNATIMP3 abolished the inhibition of the proliferation of CFs (A), the down-regulation of collagen I (B), collagen III (C), and TGF- $\beta$ (D) induced by AMO-323a-3p in the cultured CFs with the pre-treatment of Ang II (100 $\mathrm{nM})$. ( $\mathrm{n}=5$, means $\left.\pm \mathrm{SEM},{ }^{*} \mathrm{P}<0.05\right)$.

of TGF- $\beta$ induced by Ang II. Co-transfection with siRNA-TIMP3 abolished the effect of AMO$323 a-3 p$ on the expression of TGF- $\beta$. Furthermore, Co-transfection with siRNA-NC did not affect the expression of TGF- $\beta$ in the CFs with the treatment of AMO-323a-3p (Fig. 7D). These studies in vitro proved the role of miR-323a-3p/TIMP3/TGF- $\beta$ pathway in the development of cardiac fibrosis. 


\section{Cellular Physiology Cell Physiol Biochem 2018;50:2176-2187 and Biochemistry \begin{tabular}{l|l} 
DOI: 10.1159/000495059 & $\begin{array}{l}\text { Published online: } 2018 \text { The Author(s). Published by S. Karger AG, Basel } \\
\text { www.karger.com/cpb }\end{array}$
\end{tabular} \\ Zhang et al.: Inhibition of miR-323a-3p Prevented Cardiac Fibrosis}

\section{Discussion}

Cardiac fibrosis is an important hallmark of pressure overload and a main cause of decreased myocardial compliance and diverse heart diseases. Thus, therapies that contribute to inhibit cardiac fibrosis and preserve cardiac function are viewed as one of the most important goals for the treatment of heart diseases. In the current study, we focused on the effect of miR-323a-3p in the process of cardiac fibrosis and found that miR-323a-3p was upregulated in hearts subjected to TAC and rat CFs treated with Ang II. We demonstrated that miR-323a-3p induced the proliferation of CFs and enhanced the production and deposition of ECM, which resulted in cardiac fibrosis. Moreover, our data also strongly suggested that miR-323a-3p functioned as a direct regulator of TIMP3, which modulates TGF- $\beta$ expression in CFs.

Recent years, miRNA has been identified as a critical mediator in the development of fibrosis. Ge [22] found that miR-323a-3p was down-regulated in the epithelium of human and experimental murine lung fibrosis. MiR-323a-3p attenuated TGF- $\alpha$ and TGF- $\beta$ signaling by directly targeting TGFA and SMAD2. Antagomirs for miR-323a-3p augmented, and its mimics suppressed, murine lung fibrosis. Furthermore, apart from its impact on lung fibrosis, miR-323-3p has been shown to suppress the expression of SMAD2 and SMAD3 leading to inactivation of TGF- $\beta$ signaling. MiR-323-3p/TGF- $\beta$ signaling cascade was an important regulator in the metastatic progression of PDAC19. However, in this study, we found that miR-323a-3p was up-regulated in cardiac fibrosis. MiR-323a-3p promoted cardiac fibrosis by inducing CFs proliferation and TGF- $\beta$ expression. The contradictory results of these studies underline the important feature of miRNA biology, that the specific types of miRNA contribute to the biological and pathological processes of a particular organ may be tissuespecific.

As a member of the TIMP family, tissue inhibitor of matrix metalloproteinase 3 (TIMP3) can regulate many physiological effects including cell growth, hypertrophy, migration and cardiac fibrosis through MMP-dependent or MMP-independent manner [23-25]. However, whether TIMP3 is a target gene of miR-323a-3p in CFs has not been determined. In this study, we found that TIMP3 was down-regulated in hearts subjected to TAC and rat CFs treated with Ang II. We identified that TIMP3 was a target gene of miR-323a-3p, as confirmed by luciferase assay and the negative regulation of co-transfection with AMO-323a-3p, a specific inhibitor of miR-323a-3p.

TGF- $\beta$ is the most important regulator of ECM production in many organ systems and TGF- $\beta /$ Smad signaling pathway has been demonstrated to play central roles in cardiac fibrosis and in cardiac fibroblast function [26]. In our study, we observed that transfection with AM0-323a-3p significantly attenuated the up-regulation of collagen I, collagen III, MMP2, MMP9 and TGF- $\beta$ induced by Ang II in cultured CFs. AMO-323a-3p improved the cardiac function, reduced the area of fibrosis, alleviated ECM deposition and down-regulated the expression of TGF- $\beta$ in TAC mice. Additionally, co-transfection with TIMP3 siRNA could suppress the inhibition of the proliferation of CFs, the down-regulation of collagen I, collagen III and TGF- $\beta$ protein level induced by AMO-323a-3p. Hence, miR-323a-3p may function in cardiac fibroblasts by down-regulating TIMP3 expression and thus up-regulating TGF- $\beta$ activation. However, our study does not rule out the effect of Smad and other mechanisms underlying the miR-323a-3p-associated cardiac fibrosis process. Further studies are needed to evaluate.

\section{Conclusion}

In summary, our findings revealed that the inhibition of a novel pro-fibrotic miRNA, miR-323a-3p, by antagomirs was able to alleviate the cardiac fibrosis of the TAC heart and that the underlying mechanism involves an activation of the TIMP3/TGF- $\beta$ pathway. This study highlights that miR-323a-3p may be a new marker for cardiac fibrosis progression and 


\section{Cellular Physiology Cell Physiol Biochem 2018;50:2176-2187 and Biochemistry \begin{tabular}{l|l} 
DOI: 10.1159/000495059 & (c) 2018 The Author(s). Published by S. Karger AG, Basel
\end{tabular}

that inhibition of miR-323a-3p may be a promising therapeutic target for the treatment of cardiac fibrosis.

\section{Disclosure Statement}

The authors state no conflict of interests.

\section{References}

-1 Hong Y, Cao H, Wang Q, Ye J, Sui L, Feng J, Chen X: MiR-22 may suppress fibrogenesis by targeting TGFßR I in cardiac fibroblasts. Cellular physiology and biochemistry 2016;40:1345-1353.

-2 Zhang Y, Liao P, Li W, Hu D, Chen L, Guan, S: Baicalin attenuates cardiac dysfunction and myocardial remodeling in a chronic pressure-overload mice model. Cellular Physiology and Biochemistry 2017;41:849-864.

-3 Tallquist MD: Cardiac fibroblasts: from origin to injury. Current opinion in physiology 2018;1:75-79.

4 Bornstein P, Sage EH: Matricellular proteins: extracellular modulators of cell function. Current opinion in cell biology 2002;14:608-616.

5 Ambros V: The functions of animal microRNAs. Nature 2004;431:350-355.

-6 Krol J, Loedige I, Filipowicz W: The widespread regulation of microRNA biogenesis, function and decay. Nature reviews. Genetics 2010;11:597-610.

7 Du W, Liang H, Gao X, Li X, Zhang Y, Pan Z, Li C, Wang Y, Liu Y, Yuan W, Ma N, Chu W, Shan H, Lu Y: MicroRNA-328, a Potential Anti-Fibrotic Target in Cardiac Interstitial Fibrosis. Cell Physiol Biochem. 2016;39:827-836.

-8 Dai Y, Khaidakov M, Wang X: MicroRNAs involved in the regulation of postischemic cardiac fibrosis. Hypertension 2013;6:751-756.

-9 Bayoumi AS, Aonuma T, Teoh JP, Tang YL, Kim IM: Circular noncoding RNAs as potential therapies and circulating biomarkers for cardiovascular diseases. Acta pharmacologica Sinica 2018;5:22.

-10 Yuan J, Chen H, Ge D: Mir-21 Promotes Cardiac Fibrosis After Myocardial Infarction Via Targeting Smad7. Cellular physiology and biochemistry : international journal of experimental cellular physiology, biochemistry, and pharmacology 2017;42:2207-2219.

-11 Zhang C, Zhang Y, Zhu H, Hu J, Xie Z: MiR-34a/miR-93 target c-Ski to modulate the proliferaton of rat cardiac fibroblasts and extracellular matrix deposition in vivo and in vitro. Cellular signalling 2018;46:145153.

12 Shan H, Zhang Y, Lu Y: Downregulation of miR-133 and miR-590 contributes to nicotine-induced atrial remodelling in canines. Cardiovascular research 2009;83:465-472.

13 Lagendijk AK, Goumans MJ, Burkhard SB, Bakkers J: MicroRNA-23 restricts cardiac valve formation by inhibiting Has2 and extracellular hyaluronic acid production. Circulation research 2011;109:649-657.

14 Dai Y, Dai D, Mehta JL: MicroRNA-29, a mysterious regulator in myocardial fibrosis and circulating miR-29a as a biomarker. Journal of the American College of Cardiology 2014;64:2181.

-15 Pan Z, Sun X, Shan H: MicroRNA-101 inhibited postinfarct cardiac fibrosis and improved left ventricular compliance via the FBJ osteosarcoma oncogene/transforming growth factor-beta1 pathway. Circulation 2012;12:840-850.

-16 Katare R, Riu F, Mitchell K: Transplantation of human pericyte progenitor cells improves the repair of infarcted heart through activation of an angiogenic program involving micro-RNA-132. Circulation research 2011;109:894-906.

17 Wang C, Liu P, Wu H: MicroRNA-323-3p inhibits cell invasion and metastasis in pancreatic ductal adenocarcinoma via direct suppression of SMAD2 and SMAD3. Oncotarget 2016;7:14912-14924.

18 Gao Q Zheng J: microRNA-323 upregulation promotes prostate cancer growth and docetaxel resistance by repressing p73. Biomedicine \& pharmacotherapy Biomedecine \& pharmacotherapie 2018;97:528-534.

19 Yang L, Xiong Y, Hu XF, Du YH: MicroRNA-323 regulates ischemia/reperfusion injury-induced neuronal cell death by targeting BRI3. International journal of clinical and experimental pathology 2015;8:10725-10733. 


\section{Cellular Physiology Cell Physiol Biochem 2018;50:2176-2187

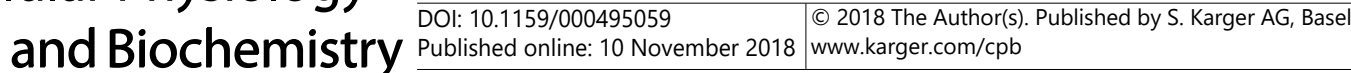 \\ Zhang et al.: Inhibition of miR-323a-3p Prevented Cardiac Fibrosis}

20 Seco-Cervera M, Gonzalez-Rodriguez D, Ibanez-Cabellos JS: Circulating miR-323-3p is a biomarker for cardiomyopathy and an indicator of phenotypic variability in Friedreich's ataxia patients. Scientific reports 2017;7:5237.

21 Pilbrow AP, Cordeddu L, Cameron VA: Circulating miR-323-3p and miR-652: candidate markers for the presence and progression of acute coronary syndromes. International journal of cardiology 2014;176:375385.

-22 Ge L, Habiel DM, Hansbro PM: miR-323a-3p regulates lung fibrosis by targeting multiple profibrotic pathways. JCI insight 2016;1:e90301.

-23 Lovelock JD, Baker AH, Gao F: Heterogeneous effects of tissue inhibitors of matrix metalloproteinases on cardiac fibroblasts. American journal of physiology. Heart and circulatory physiology 2005;288:H461-468.

24 Moore L, Fan D, Basu R, Kandalam V, Kassiri Z: Tissue inhibitor of metalloproteinases (TIMPs) in heart failure. Heart failure reviews 2012;17:693-706.

25 Fan D, Takawale A, Basu R: Differential role of TIMP2 and TIMP3 in cardiac hypertrophy, fibrosis, and diastolic dysfunction. Cardiovascular research 2014;103:268-280.

-26 MacLean J, Pasumarthi KB: Signaling mechanisms regulating fibroblast activation, phenoconversion and fibrosis in the heart. Indian journal of biochemistry \& biophysics 2014;51:476-482. 